\title{
Theoretical approach to one-dimensional detonation instability*
}

\author{
Chun WANG $^{\dagger}$, Gaoxiang XIANG, Zonglin JIANG \\ State Key Laboratory of High Temperature Gas Dynamics, Chinese Academy of Sciences, \\ Beijing 100190, China
}

\begin{abstract}
Detonation instability is a fundamental problem for understanding the microbehavior of a detonation front. With the theoretical approach of shock dynamics, detonation instability can be mathematically described as a second-order ordinary difference equation. A one-dimensional detonation wave can be modelled as a type of oscillators. There are two different physical mechanisms controlling the behaviors of a detonation. If the shock Mach number is smaller than the equilibrium Mach number, the fluid will reach the sonic speed before the end of the chemical reaction. Then, thermal chock occurs, and the leading shock becomes stronger. If the shock Mach number is larger than the equilibrium Mach number, the fluid will be subsonic at the end of the chemical reaction. Then, the downstream rarefaction waves propagate upstream, and weaken the leading shock. The above two mechanisms are the basic recovery forces toward the equilibrium state for detonation sustenance and propagation. The detonation oscillator concept is helpful for understanding the oscillating and periodic behaviors of detonation waves. The shock dynamics theory of detonation instability gives a description of the feedback regime of the chemical reaction, which causes variations of the leading shock of the detonation.
\end{abstract}

Key words detonation wave, detonation instability, shock wave, chemical reaction

Chinese Library Classification O362, O381, O354.5

2010 Mathematics Subject Classification 80A25, 39A11, 76L05

\section{Nomenclature}

a, sonic speed;

$E_{\text {a }}, \quad$ chemical activation energy;

$k$, chemical rate factor;

$L_{\mathrm{CJ}}$ reaction zone length in the ChapmanJouguet (CJ) state;

$M, \quad$ moving Mach number of leading shock;

$M_{\mathrm{e}}$, equilibrium Mach number of leading shock;

$p$, pressure;

$Q$, chemical heat;

$R, \quad$ gas constant;

$S$, chemical reaction source term; $t, \quad$ time;

u, velocity;

$x$ distance from shock position to its equilibrium state;

$\rho, \quad$ density;

$\gamma, \quad$ specific heat ratio;

$\lambda, \quad$ chemical reaction progress;

$\mu, \quad$ function of $M$ and $\gamma$;

$\omega$, chemical reaction rate;

$\nu$ reaction order.

\footnotetext{
* Received Nov. 13, 2015 / Revised Feb. 4, 2016

Project supported by the National Natural Science Foundation of China (No.90916028) and the Innovation Program of the State Key Laboratory of High Temperature Gas Dynamics of Institute of Mechanics, Chinese Academy of Sciences

$\dagger$ Corresponding author, E-mail: wangchun@imech.ac.cn
} 


\section{Introduction}

Detonation is a distinctive phenomenon in physics. It is related to high-pressure explosion, strong combustion, thermonuclear supernovae, etc. Detonation can occur in conventional solid and liquid explosives, reactive gases, and interstellar media in cosmology ${ }^{[1-4]}$. It propagates with high speed and high pressure, involving the coupling of shock waves, reactions, and turbulence. The most well-known theories describing detonation are the Chapman-Jouguet (CJ) theory and the Zeldovich-von Neuman-Doring (ZND) model. Detonation waves have special selforganized micro-structures, which occur due to the detonation instability such as the movement of the transverse waves in the detonation front and the cellular structure recorded after the detonation propagation ${ }^{[5-8]}$. An excellent experimental study on cellular detonation was made by Pintgen et al..$^{[9]}$, which revealed clear transverse waves in the detonation front by use of planar laser-induced fluorescence (PLIF). Unstable detonation has attracted increasing interest from researchers because it is thought to be related to the detonation limit, the critical energy of detonation initiation, and the critical diameters of detonation propagations. The problem of detonation instability concerns the mechanisms of multi-dimensional detonation, unstable detonation, one-dimensional pulsating detonation, etc. ${ }^{[10-12]}$.

Until now, the mechanism of detonation instability has not been well understood. In addition to the experimental studies on unstable detonation and multi-dimensional detonation, there have been two approaches to the problem of detonation instability. One is theoretical analyses, and the other is numerical simulation. Erpenbeck ${ }^{[13-14]}$ gave the earliest theoretical hydrodynamic stability analysis of a detonation wave by use of a Laplace transformation on the linearized reactive Euler equation, and obtained an initial value. The method of Erpenbeck utilizes a small-perturbation analysis for a linear stability problem, and is primarily concerned with the development of small perturbations with time evolution. Later, Lee and Stewart ${ }^{[15]}$ presented a normal-mode approach to the problem of linear detonation stability. They analyzed the behavior of small perturbations in the steady ZND detonation in the shock frame, considering the growth rate of the small perturbation waves, the frequency eigenvalue, and the wave number. Fickett and Wood ${ }^{[16]}$ numerically found the periodic oscillating behaviors of one-dimensional detonation, called pulsating detonation. Abouseif and Toong ${ }^{[17]}$ studied onedimensional pulsating detonation with different activation energies and over-driven degrees, and found that, in the case of high activation energy, the oscillations of detonation transformed to irregular periodic modes in which high-frequency oscillations existed. Numerical studies further showed that there were non-equilibrium behaviors in the detonation initiation and collisions of the detonations with a head-on shock wave. Lee and Higgins ${ }^{[18]}$ gave three mechanisms of detonation initiation with different initiation energies, i.e., supercritical initiation, critical initiation, and sub-critical initiation. $\mathrm{Ng}$ and Lee ${ }^{[19]}$ conducted a numerical study of detonation initiation by use of a three-step chain-branching chemical kinetic model, and showed that different oscillation modes existed in the detonation initiations. Han et al. ${ }^{[20]}$ numerically studied the behaviors of detonation when the detonation collided with a head-on shock wave, and found that, after the collision, detonation decoupled first, then accelerated to over driven detonation, and finally decayed to the detonation propagating at the CJ speed.

The multi-dimensional structures of the detonation produced in the above numerical studies of multi-dimensional detonation are by solving the reactive Euler equations. It is important to note that numerical simulations have provided a deeper understanding of detonation instability, with which the main factors can be studied individually and the effects of the complex physical phenomena can be decoupled ${ }^{[21-23]}$. Kasimov and Stewart ${ }^{[24]}$ presented an excellent study on the dynamics analysis of self-sustained one-dimensional detonation by use of a characteristic line equation and several selected selecting precise non-reflection boundary conditions, studied the detonation behavior on the shock frame, and presented the periodic dynamic features of the leading shock and sonic plane. Zhang et al. ${ }^{[25]}$ proposed a detonation oscillator concept 
analogy to the Helmholtz oscillator, which could be used to understand the periodic oscillating behavior of one-dimensional detonation with regular and irregular cycles. However, the physical mechanism of the detonation oscillation was not clarified.

In this paper, the theoretic approach to the detonation oscillator from the shock dynamics in reactive media is expected to build the theoretical model for the detonation instability. Different control mechanisms of the detonation oscillation, e.g., the role of chemical reactions in under-driven conditions, are expected to be clarified.

\section{Theoretical modelling of one-dimensional pulsating detonation}

In this paper, an approach to shock dynamics in reactive flow will be developed to model the interaction of shock waves and chemical reactions. The physical model is simply presented in Fig. 1 (in the shock frame), where a moving shock propagates in a reactive medium, the temperature and pressure are raised just behind the shock wave, and a chemical reaction is induced. The subsonic flow compressed by the shock wave is accelerated by a release of chemical heat. Once the subsonic flow has been accelerated to the sonic speed, an oscillator will automatically form. In the following section, we will give a theoretical proof to this oscillator model by use of the method of shock dynamics in the reactive flow.

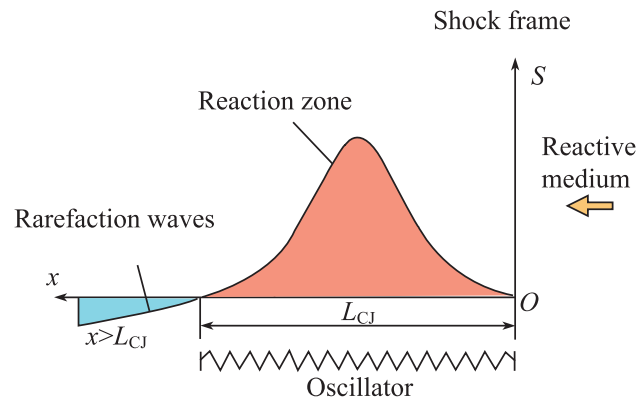

Fig. 1 Detonation oscillator concept for one-dimensional detonation instability

Among the methods used to deduce the Chisnell-Chester-Witham (CCW) relations (the basics of shock dynamics), the method of Witham is the simplest ${ }^{[26-27]}$. To study the basic mechanism of the combustion oscillation induced by the shock wave and chemical reaction, the shock dynamics in the chemical reaction flow following the method of Witham are developed here. For convenience, the following right-travelling characteristic line equation is considered:

$$
\frac{\mathrm{d} p}{\mathrm{~d} t}+\rho u \frac{\mathrm{d} u}{\mathrm{~d} t}-(\gamma-1) Q S=0 .
$$

For the moving shock, the relations of the gas parameters across the shock wave follow the following difference equation:

$$
\mathrm{d} p_{2}=\frac{4 \gamma p_{1}}{\gamma+1} M \mathrm{~d} M, \quad \mathrm{~d} u_{2}=\frac{2 a_{1}}{\gamma+1}\left(1+\frac{1}{M}\right) \mathrm{d} M, \quad \rho_{2} u_{2}=\frac{\rho_{1} a_{1} M}{\mu},
$$

where the subscripts 1 and 2 denote the gas states just in front of the shock wave and just behind the shock wave, respectively. $M$ is the moving Mach number of the shock wave, and

$$
\mu=\left(\frac{(\gamma-1) M^{2}+2}{2 \gamma M^{2}-(\gamma-1)}\right)^{\frac{1}{2}}
$$

Substituting Eq. (2) into Eq. (1) yields the following shock dynamic equation for a moving shock with chemical reactions in a reactive flow:

$$
\frac{2 M}{\gamma+1}\left(2+\frac{1}{\mu}\left(1+\frac{1}{M^{2}}\right)\right) \frac{\mathrm{d} M}{\mathrm{~d} t}-\frac{(\gamma-1) Q S}{\rho_{1} a_{1}^{2}}=0 .
$$


It describes the behaviors of the moving shock with different chemical reactions. When $S=0$, $\frac{\mathrm{d} M}{\mathrm{~d} t}=0$, which means that, when the Mach number of the moving shock does not change, the propagation speed of the shock wave will be constant. There are two cases matching the condition $S=0$. One is the case without any chemical reaction. It indicates a free-propagating shock wave in a non-reactive medium. The other case is with a chemical reaction, but the chemical reaction rate is equal to zero at the end of the chemical reaction. In the case with a chemical reaction, if the fluid velocity just reaches the sonic speed, the chemical reaction zone will not be affected by the downstream flow conditions, i.e., there will be an equilibrium state for the moving shock that is self-sustained. Typically, this equilibrium state is a special state because it fully matches the CJ conditions of detonation. In other words, for a onedimensional self-sustained shock wave supported by a chemical reaction, the equilibrium state corresponds to a detonation wave propagating with the CJ velocity. Equation (3) indicates that for the moving shock propagating in a reactive media, the strength of the moving shock will be directly controlled by the chemical reaction progress. Specifically, in the states near the equilibrium, the variations in the chemical reaction will (inevitably) cause the instability of the moving shock. For a moving shock coupled with a chemical reaction, there is an equilibrium state, in which the gaseous fluid reaches the sonic speed at the end of the chemical reaction. When the chemical reaction varies around the equilibrium state, the strength of the leading shock will vary around the equilibrium shock speed, which corresponds to the CJ detonation. Near the equilibrium state, Eq. (3) can be rewritten as follows:

$$
\begin{aligned}
& f(M) \frac{\mathrm{d}\left(M-M_{\mathrm{e}}\right) a_{1}}{\mathrm{~d} t}-\frac{(\gamma-1) Q S}{\rho_{1} a_{1}}=0, \\
& f(M)=\frac{2 M}{(\gamma+1)}\left(2+\frac{1}{\mu}\left(1+\frac{1}{M^{2}}\right)\right),
\end{aligned}
$$

where $M_{\mathrm{e}}$ is the Mach number of the shock wave in the equilibrium state, i.e., the CJ Mach number of detonation.

We select the inertial coordinate system as $x_{\mathrm{e}}=M_{\mathrm{e}} a_{1} t$, and let $x=x_{\mathrm{s}}-x_{\mathrm{e}}$, where $x_{\mathrm{s}}$ is the shock position in the laboratory coordinate, and $M$ is the shock Mach number in the laboratory coordinate. Define the chemical reaction source term as a function of the spatial order with the quasi-steady ZND model, i.e., $S=S(x, M)$. Then, Eq. (3) can be reexpressed as follows:

$$
f(M) \frac{\mathrm{d}^{2} x}{\mathrm{~d} t^{2}}-\frac{(\gamma-1) Q S(x, M)}{\rho_{1} a_{1}}=0 .
$$

The physical meaning of $x$ is the distance from the shock position to its assumed position in the equilibrium state. It is a spatial parameter for the shock wave oscillation. Equation (6) is a second-order ordinary difference equation, which means that the interaction of the moving shock wave and the chemical reaction can be modelled as a kind of oscillators and the variations of the leading shock are controlled primarily by the variations in the chemical reaction. Because of the nonlinearity of functions $f(M)$ and $S(x, M)$, the oscillator is a nonlinear oscillator. The typical source term $S(x, M)$ is the chemical reaction rate in the sonic plane behind the leading shock. The above oscillator can be physically described as follows: in the case of a chemical reaction induced by the shock wave, when the gas flow is heated to the sonic speed by the chemical reaction, then the oscillator will be bounded by the leading shock and the sonic plane will automatically form. With the perturbation of the chemical reaction around the sonic plane, the shock speed will oscillate with a certain period.

\section{Mechanism for detonation oscillation}

Figure 2 presents a numerical example of pulsating the detonation with the one-step chemical reaction model. The non-dimensional activation energy is $\bar{E}_{\mathrm{a}}=\frac{E_{\mathrm{a}}}{\rho_{\mathrm{s}} a_{\mathrm{s}}^{2}}=13.9$, where $\rho_{\mathrm{s}}$ and $a_{\mathrm{s}}$ 
are the gaseous density and the sound speed just behind the leading shock wave, respectively. The peak pressure $p_{\max }$ is the maximum local pressure during the detonation propagation. From Fig. 2, we find that the peak pressure simply oscillates around its equilibrium CJ value.

In the red region (up half region), over-driven detonation exists, where a larger peak pressure than the CJ value occurs. In the blue region (down half region), under-driven detonation exists, where a lower peak pressure than the CJ value occurs. For over-driven detonation, rarefaction waves are the dominant mechanism for the detonation decay. For under-driven detonation, chemical reactions are the dominant mechanism for the detonation acceleration. These two mechanisms cause the detonation to oscillate around its equilibrium state.

There are two necessary conditions for the existence of the oscillator induced by the interaction of the shock wave and the chemical reaction, i.e., the subsonic flow derives from the shock compression and the subsonic flow is accelerated to the sonic speed by the chemical heat release. In a periodic cycle of shock wave oscillation, there are two different physical mechanisms controlling the variation of the shock wave speed.

Figure 3 shows the flow variables of the reaction zone in the situations of under-driven detonation and over-driven detonation with a one-step chemical reaction ${ }^{[28]}$, i.e.,

$$
\omega=k(1-\lambda)^{\nu} \mathrm{e}^{\frac{E_{\mathrm{a}}}{R T}},
$$

where $\omega$ is the Arrhenius reaction rate with the activation energy $E_{\text {a }}$, the reaction order $\nu$, and the pre-exponent $k, R$ is the universal gas constant, and $T$ is temperature.

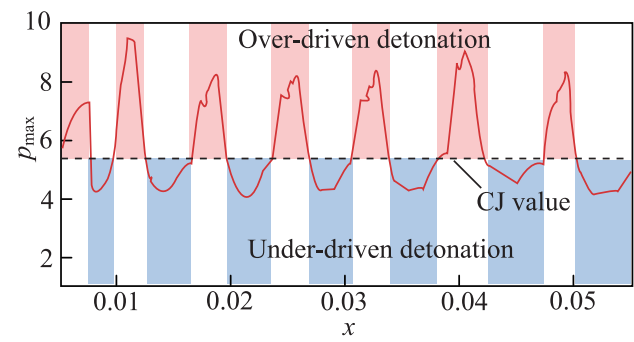

Fig. 2 Numerical example of shock pressure during detonation propagation

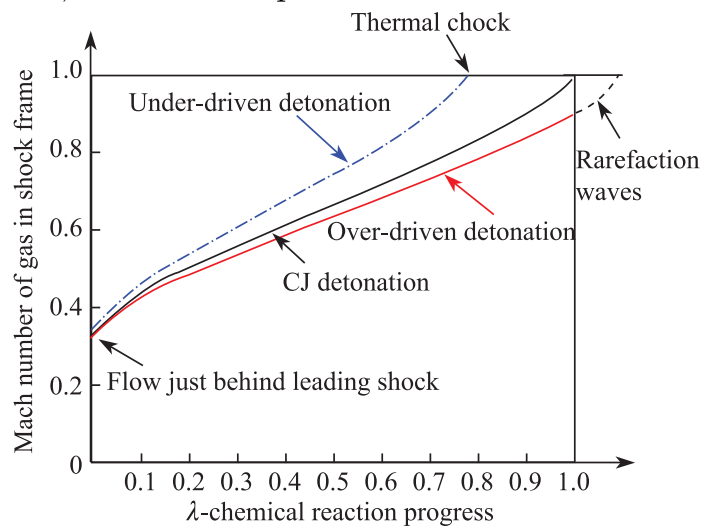

Fig. 3 Flow variables of reaction zone in different detonation conditions

From Fig. 3, we can see that, if the shock Mach number is lower than the equilibrium Mach number, the fluid will reach the sonic speed before the end of the chemical reaction, and thermal chock takes place, which increases the strength of the shock wave; if the shock Mach number is larger than the equilibrium Mach number, the fluid is subsonic at the end of the chemical reaction, and thus the downstream rarefaction waves will propagate upstream and weaken the shock wave. The above two mechanisms are the basic recovery forces toward the equilibrium state for the oscillation of a shock wave coupled with a chemical reaction. It is clear that this oscillator (induced by a shock wave and the chemical reaction interaction) is a self-excited oscillator. Typically, there is a spatial distance between the leading shock and the sonic plane, which is defined as the reaction zone length. If the shock Mach number is lower than the equilibrium Mach number, the reaction zone length will be shorter than that in the equilibrium state. The reaction zone length has a tendency to approach the equilibrium length, which causes the shock Mach number to be larger than the equilibrium Mach number. The CJ theory considers detonation as a discontinuity. The ZND model gives a quasi-stationary description of one-dimensional detonation. The oscillator model is a high order description of the unstable detonation wave. Moreover, such an oscillator model can be used to explain the overdriven 
phenomena in the transition from deflagration to detonation and the detonation relaxation in the process of detonation colliding with a weak head-on shock wave.

There have been several studies of the detonation instability concerning the unstable detonation patterns of over-driven detonation. In fact, the behaviors of over-driven detonation can be controlled by the rarefaction waves behind the detonation, whose effects on the detonation front are nearly linear, especially in weak over-driven detonation. The effects of the chemical reaction on under-driven detonation are more important to the nonlinear behaviors of propagating detonation. Unfinished nonlinear chemical reactions have faster and stronger effects on the acceleration behaviors of the detonation front of a detonation oscillator, which may be enhanced by the combustion instability and turbulence in the reaction zone.

Figures 4(a) and 4(b) present the distance $x$ from the leading shock of detonation to the sonic plane in the under-driven detonation conditions. This distance can be treated as the length of the detonation oscillator in the under-driven detonation condition. The variable $x$ is both nonlinear and non-monotonic. At the low non-dimensional activation energy, e.g., $\bar{E}_{\mathrm{a}}=10$, the range of the single solution zone around the CJ state of detonation is relatively wide. Therefore, the oscillation detonation tends to be regular and single-periodic. At the high non-dimensional activation energy, e.g., $\bar{E}_{\mathrm{a}}=50$, the range of the single solution zone around the CJ state of detonation is narrow and the double solution zone is dominant, resulting in period bifurcation and irregular and multi-periodic oscillation detonation.

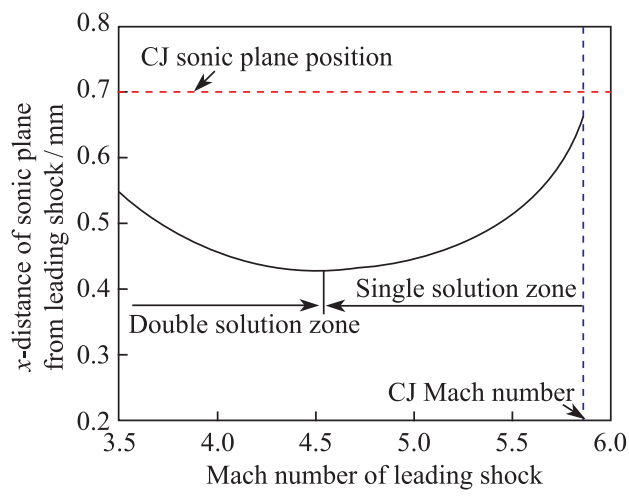

(a) $\bar{E}_{\mathrm{a}}=10$

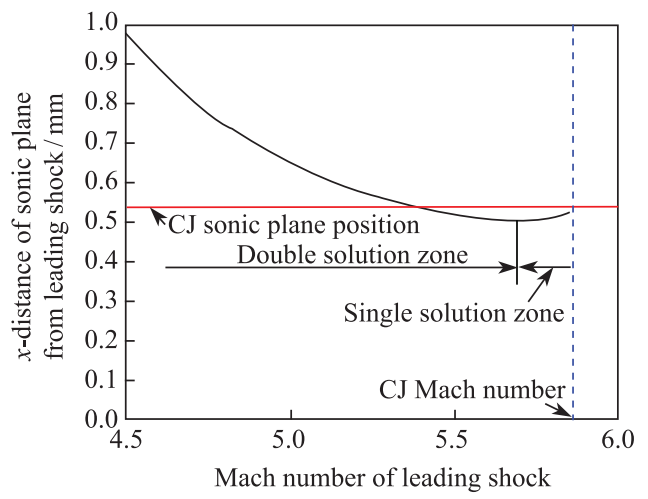

(b) $\bar{E}_{\mathrm{a}}=50$

Fig. 4 Distance $x$ from leading shock of detonation to sonic plane in under-driven detonation

From the above analysis, we find that the shock dynamics of detonation instability gives a simple description of the feedback regime of the chemical reaction, resulting in the variation of the leading shock of detonation. In the under-driven detonation, the unfinished chemical reaction and thermal chock generate perturbations propagating upstream, and enhance the leading shockwave. Because of the chemical reaction nonlinearity, the length of the chemical reaction zone in the detonation front is nonlinear and non-monotonic, resulting in the complex oscillating behaviors of the detonation oscillator. The chemical reaction model and the activation energy of the chemical reaction are the key factors for the behaviors of unstable propagating detonation waves.

\section{Mechanism of multi-dimensional cellular detonation}

In real three-dimensional detonation, the curvature variation of the detonation front and turbulent reaction play important roles in the detonation instability. Typically, the concave front of the detonation strengthens the reaction, and the convex front of the detonation weakens the reaction. The turbulence always enhances the reaction in the reaction zone of the detonation. There is competition between the curvature of the detonation front and the chemical reaction 
in the detonation front. A numerical cellular detonation with lower activation energy in the two-dimensional situation is shown in Fig. 5. The chemical reaction zone can be considered as a two-dimensional oscillator, where the curvature of the leading shock wave is an important factor for the multi-dimensional detonation propagations, and the detonation always tends toward the equilibrium state determined by the above factors. The detonation oscillator concept is helpful for understanding the oscillating and periodic behaviors of the detonation waves.

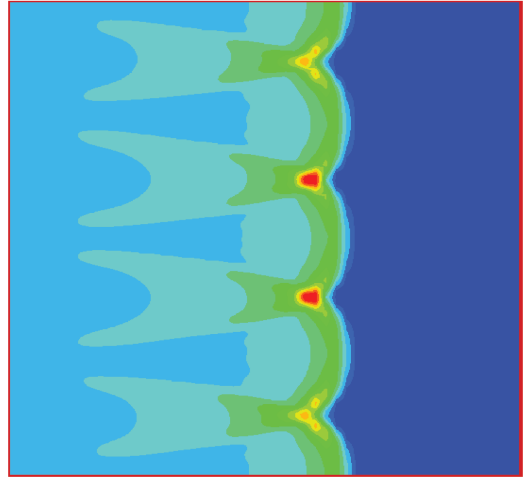

(a) Transverse waves and pressure contours

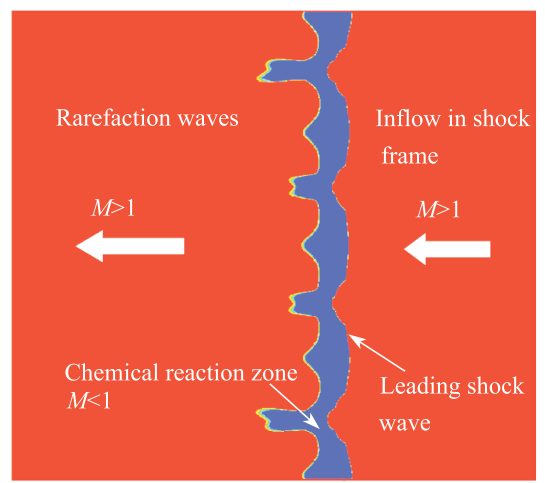

(b) Subsonic reaction zone in shock frame

Fig. 5 Numerical example of two-dimensional regular detonation

\section{Conclusions}

Since the detonation instability can be mathematically described as a second-order ordinary difference equation by the theoretical approach of shock dynamics, one-dimensional pulsating detonation can be modelled as a type of oscillators. In a periodic cycle of a shock wave oscillation, there are two different physical mechanisms controlling the variation of the shock wave speed. If the shock Mach number is lower than the equilibrium Mach number, the fluid will reach the sonic speed before the end of the chemical reaction, and "thermal chock" will take place, which strengthens the shock wave. If the shock Mach number is larger than the equilibrium Mach number, the fluid will be subsonic at the end of the chemical reaction, and thus the downstream rarefaction waves will propagate upstream and weaken the shock wave. The above two mechanisms are the basic recovery forces toward the equilibrium state for the oscillation of a shockwave coupled with a chemical reaction. This oscillator (induced by the shock wave and chemical reaction interaction) is a self-excited oscillator. The detonation oscillator concept is helpful for understanding the oscillating and periodic behaviors of the detonation waves. The shock dynamics theory of detonation instability gives a description of the feedback regime of the chemical reaction causing the variation of the leading shock of the detonation.

\section{References}

[1] Gulhan, A. and Beylich, A. E. Detonation wave phenomena in bubbled liquid. AIP Conference Proceedings, 208, 825-829 (1990)

[2] Petrone, F. J. Validity of the classical detonation wave structure for condensed explosives. Physics of Fluids, 11, 1473-1478 (1968)

[3] Noeel, C., Busegnies, Y., Papalexandris, M. V., and Goriely, S. Hydrodynamical simulations of detonations in superbursts. AIP Conference Proceedings, 1016, 418-420 (2008)

[4] Larson, D. B. and Sisemore, C. J. A peak stress gauge for determining the yield of underground nuclear detonations. Journal of Applied Physics, 39, 5609-5612 (1968)

[5] Duff, R. E. Investigation of spinning detonation and detonation stability. Physics of Fluids, 4, 1427-1433 (1961) 
[6] Wang, C., Jiang, Z. L., and Gao, Y. L. Half-cell law of regular cellular detonation. Chinese Physics Letters, 25, 3704-3707 (2008)

[7] Wang, C., Jiang, Z. L., Hu, Z. M., and Han, G. L. Numerical investigation on evolution of cylindrical cellular detonation. Applied Mathematics and Mechanics (English Edition), 29(11), 1487-1494 (2008) DOI 10.1007/s10483-008-1109-y

[8] Jiang, Z. L., Han, G. L., Wang, C., and Zhang, F. Self-organized generation of transverse waves in diverging cylindrical detonations. Combustion and Flame, 156, 1653-1661 (2009)

[9] Pintgen, F., Eckett, C., Austin, J., and Shepherd, J. Direct observations of reaction zone structure in propagating detonations. Combustion and Flame, 133, 211-229 (2003)

[10] Ng, H. D. and Zhang, F. Shock Wave Science and Technology Reference Library, Springer-Verlag, Heidelberg, 107-212 (2012)

[11] Lee, J. H. S. Dynamic parameters of gaseous detonations. Annual Review of Fluid Mechanics, 16, 311-336 (1982)

[12] Abouseif, G. and Toong, T. Theory of unstable detonation. Combustion and Flame, 45, 67-94 (1982)

[13] Erpenbeck, J. J. Stability of steady-state equilibrium detonations. Physics of Fluids, 5, 604-614 (1962)

[14] Erpenbeck, J. J. Stability of idealized one reaction detonations. Physics of Fluids, 7, 684-696 (1964)

[15] Lee, H. and Stewart, D. Calculation of linear detonation instability: one-dimensional instability of planar detonation. Journal of Fluid Mechanics, 216, 103-132 (1990)

[16] Fickett, W. and Wood, W. W. Flow calculations for pulsating one-dimensional detonations. Physics of Fluids, 9, 903-916 (1966)

[17] Abouseif, G. and Toong, T. Theory of unstable detonation. Combustion and Flame, 45, 67-94 (1982)

[18] Lee, J. H. S. and Higgins, A. Comments on criteria for direct initiation of detonation. Philosophical Transactions of the Royal Society, A: Mathematical, Physical and Engineering Sciences, 357, 3503-3521 (1999)

[19] Ng, H. D. and Lee, J. H. S. Direct initiation of detonation with a multi-step reaction scheme. Journal of Fluid Mechanics, 476, 179-211 (2003)

[20] Han, G. L., Jiang, Z. L., and Zhang, D. L. Numerical investigation on the collision between detonations and shocks (in Chinese). Chinese Journal of Theoretical and Applied Mechanics, 40, 154-161 (2008)

[21] Yuan, Y. W. An approximate analytical solution of the cylindrical detonation wave generated by the linear explosion. Applied Mathematics and Mechanics (English Edition), 14(5), 437-440 (1993) DOI 10.1007/BF02453764

[22] Guo, Y. H., Tian, Z., and Hao, B. T. Implicit TVD schemes applied to gas-droplet detonation calculation. Applied Mathematics and Mechanics (English Edition), 21(6), 725-732 (2000) DOI $10.1007 / \mathrm{BF} 02460192$

[23] Liu, Y. J. and Sheng, W. C. Generalized Riemann problem for gas dynamic combustion. Applied Mathematics and Mechanics (English Edition), 32(8), 1079-1089 (2011) DOI 10.1007/s10483-0111482-6

[24] Kasimov, A. R. and Stewart, D. S. On the dynamics of selfsustained one-dimensional detonations: a numerical study in the shock-attached frame. Physics of Fluids, 16, 3566-3578 (2004)

[25] Zhang, F., Chue, R., Lee, J. H. S., and Klein, R. A nonlinear oscillator concept for one-dimensional pulsating detonations. Shock Waves, 8, 351-359 (1998)

[26] Han, Z. Y. and Yin, X. Z. Shock Dynamics, Kluwer Academic Publishers Group, Netherlands (1998)

[27] Witham, G. On the propagation of shock waves through regions of non-uniform area or flow. Journal of Fluid Mechanics, 84, 337-366 (1958)

[28] Kasimov, A. R. and Stewart, D. S. On the dynamics of self-sustained one-dimensional detonations: a numerical study in the shock-attached frame. Physics of Fluids, 16, 3566-3578 (2004) 